\section{Christoph Bachmann}

\section{Tibetische Medizin}

Das herrschende Prinzip unserer Schulmedizin sucht bei einer Erkrankung immer eine Störung, d.h. eine krankhafte Veränderung von Gewebe oder einer Organfunktion, die mit einem Medikament oder einer nichtmedikamentösen Behandlung wieder in den gesunden Zustand zurückgeführt wird. Und auch die Phytotherapie nach westlicher Richtung richtet sich oft nach diesem schulmedizinischen Denken. In der Tibetischen Medizin besteht eine Krankheit nicht primär in einer Gewebeveränderung oder Fehlfunktion eines Organs, sondern in einem Ungleichgewicht der drei dynamischen Zustände rLung (Wind: das bewegende Prinzip), Tripa (Galle: das wärmende Prinzip) und Beken (Schleim: das kühlende bzw. stabilisierende Prinzip), die normalerweise ein Gleichgewicht bilden und so für ein einwandfreies Funktionieren des Körpers und des Geistes sorgen.

Besteht ein solches Ungleichgewicht und zeigt es sich in körperlichen oder mentalen Symptomen, sucht der tibetische Arzt nach einer Möglichkeit, Gegengewicht zu geben und das Gleichgewicht wiederherzustellen. Dies geschieht, indem der Patient Anweisungen erhält, sein Verhalten, seine Gewohnheiten und seine Ernährung zu verändern. Reicht dies nicht aus, setzt der Arzt ein pflanzliches Vielstoffgemisch ein, das zum Ziel führen kann.

\title{
Die tibetische Rezeptur Se'bru 5 bei rezidivierenden unkomplizierten Harnwegsinfekten: Eine Fallserie zeigt Wirksamkeit
}

Das tibetische Arzneimittel Se'Bru 5 (Padma Digestin ${ }^{\circledR}$ ), wie die meisten tibetischen Arzneimittel ein pflanzliches Vielstoffgemisch, ist in der Schweiz zur Behandlung von Verdauungsstörungen sowie von Appetitmangel zugelassen. Seine Wirksamkeit wurde mit einer klinischen Studie dokumentiert. Im folgenden Beitrag wird diese aufgezeigt sowie ein Fallbericht zur Anwendung gemäss den Prinzipien der Tibetischen Medizin bei Frauen mit rekurrierenden Zystitiden vorgestellt.

\section{Tibetische Arzneimittel}

Tibetische Arzneimittel sind meistens pflanzliche Vielstoffgemische, die aus 5-20 getrockneten und gemahlenen Arzneipflanzen bestehen. Zum Teil enthalten diese Gemische auch noch Mineralien. Solche Vielstoffgemische üben eine synergistische Wirkung aus. Deshalb ist von den einzelnen Inhaltsstoffen eine wesentlich geringere Dosis zur Entfaltung der Arzneimittelwirkung notwendig, als dies erforderlich wäre, wenn eine Monosubstanz diese Wirkung erzielen müsste.

\section{Schnittstelle}

Durch verschiedene historische Umstände wurde die Tibetische Medizin in den Westen gebracht, wo sie mit den Methoden der modernen Medizin untersucht wurde. Es gibt klinische Untersuchungen, welche die Wirksamkeit tibetischer Arzneimittel belegen (siehe unten). In der Schweiz werden einige tibetische Medikamente gemäss den strengen Vorschriften für Arzneimittel hergestellt.
Kasten 1. Se'bru 5-Kapseln

- Zusammensetzung: getrocknete und gemahlene
- Granatapfelsamen (204 g),
- Galgantwurzel (102 g),
- Früchte des Langen Pfeffers (25,5 g),
- Kardomensamen ( $12,75 \mathrm{~g})$,
- Kassia-Zimtrinde (12,75 g).
- Das Medikament hat in der Schweiz die Zulas-
sung für die Indikationen Verdauungsstörun-
gen sowie Appetitmangel (Markenname in der
Schweiz: Padma Digestin (Zulassungsnummer
59375 Swissmedic)).

Durch diese tibetische Tradition, die moderne Arzneimittelforschung und die hohe Herstellungsqualität können solche tibetische Medikamente als Schnittstelle zwischen Ethnomedizin, Phytotherapie und Schulmedizin bezeichnet werden.

\section{Se'bru 5}

Das tibetische Medikament Se'Bru 5 besteht aus 5 verschiedenen Arzneipflanzen, die zum Teil auch in der westlichen Phytotherapie verwendet werden (Kasten 1).

Gemäss der tibetischen Tradition bilden die 5 Arzneipflanzen ein wär-

\section{KARGER}

(C) 2017 S. Karger GmbH, Freiburg

Fax +497614520714 
mendes Prinzip, das die Verdauungswärme medrod (gesprochen: Metö) stärkt. Unteraspekte der drei oben genannten Prinzipien rLung, Tripa und Beken regulieren den Verdauungsvorgang. Sie vermischen die Nahrungsbestandteile mit Wasser und zersetzen sie, damit der eigentliche Verdauungsvorgang stattfinden kann. Sie setzen die Magenmotilität in Gang und verdauen feste und flüssige Nahrung.

\section{Wirksamkeit bei funktioneller Dyspepsie}

Die klinische Wirksamkeit von Se'bru 5 bei Verdauungsbeschwerden wurde in einer klinischen Studie nach westlichen Massstäben nachgewiesen [1]. Bei dieser offenen, prospektiven klinischen Studie wurden 37 Patienten rekrutiert, die in verschiedenen ärztlichen Privatpraxen behandelt wurden. Diese Patienten litten seit mindestens 4 Wochen unter dyspeptischen Beschwerden, die sich mindestens einmal pro Woche mit mindestens zwei typischen dyspeptischen Symptomen manifestierten. Die Behandlung dauerte 5 Wochen, die tägliche Dosis betrug zweimal 3 Kapseln. Die Symptome wurden bei Baseline, nach 3 Wochen, am Schluss der Studie sowie nach weiteren 4 Wochen überprüft.

Die Wirksamkeit von Se'Bru 5 wurde mit dem Digest-Fragebogen ermittelt, der das Vorhandensein und den Schweregrad verschiedener dyspeptischer Beschwerden wie postprandiales Völlegefühl, diffuse epigastrische Beschwerden, Regurgitation, Erbrechen, vorzeitiges Sättigungsgefühl, Unterbauchbeschwerden usw. dokumentiert. Sowohl die Prüfärzte als auch die Patienten beurteilten getrennt die Wirksamkeit des Präparates. Schliesslich wurden noch Parameter der Lebensqualität (quality of life (QOL)) erhoben.

Von den 37 rekrutierten Patienten konnten 22 in die Per-Protokoll-Aus- wertung eingeschlossen werden. Die Behandlung führte $\mathrm{zu}$ einer signifikanten Verbesserung der Häufigkeit und des Schweregrades der dyspeptischen Beschwerden $(p<0,01)$. Eine Signifikanz wurde bei den folgenden Symptomen beobachtet:

- postprandiales Völlegefühl,

- Nausea,

- epigastrische Beschwerden/ Schmerzen,

- Magenkrämpfe,

- Appetitmangel.

Ein Trend zur Verbesserung zeigte sich bei:

- frühzeitigem Sättigungsgefühl,

- Blähungen,

- Verstopfung,

- nächtlichen Schmerzen bzw.

Schmerzen bei Nüchternheit.

Der Beginn der Verbesserung wurde durchschnittlich nach 7 Tagen, das Verschwinden nach durchschnittlich 22 Tagen ermittelt.

Die QOL verbesserte sich im Schnitt hochsignifikant $(p<0,001)$.

Die Verträglichkeit des Präparates wurde von Prüfärzten überwiegend (>80\%) als gut bis sehr gut bezeichnet.

\section{Rezidivierende Zystitis}

Gemäss der Tibetischen Medizin ist die Verdauungswärme medrod jedoch nicht nur für die Verdauungsfunktionen wichtig, sondern für alle Organe und Funktionen im Abdomen. Ein zu schwach ausgeprägtes medrod kann sich im sogenannten «Kalte-NierenSyndrom» äussern, worunter unter anderem z.B. Fertilitäts- und Libidoverminderung und auch rezidivierende Zystitiden fallen. In einem Journal für Tibetische Medizin wurde vor Kurzem eine Fallstudie publiziert, welche die Möglichkeit einer neuen Indikation für Se'Bru 5 vorstellt [2]. Es handelt sich dabei um eine Off-labelIndikation, weil das Präparat, wie erwähnt, in der Schweiz nur die Zulassung für die Indikationen «Verdauungsstörungen sowie Appetitmangel» erhalten hat.
Die Autoren dieses Artikels beschreiben die Behandlung von 5 weiblichen Patienten mit Tibetischer Medizin, die seit mindestens 2 Jahren an chronischen, unkomplizierten Harnwegsinfekten litten, welche sich mit mindestens 3 Infektionen pro Jahr äusserten. Die Patientinnen wurden früher üblicherweise mit Antibiotika behandelt. Eine Patientin hatte schon eine Therapie mit Probiotika versucht, aber keine nahm Cranberry-Saft, eine beliebte komplementärmedizinische Behandlungsart von Harnwegsinfekten, ein.

\section{Behandlung}

Die Patientinnen nahmen während 6 Monaten jeden Morgen 2 Kapseln Se'Bru 5 ein.

Weiter erhielten sie folgende, mit der Tibetischen Medizin übereinstimmende Diät- und Verhaltensanweisungen:

- Reduktion oder Vermeiden von Nahrungsmitteln, die Beken, also das kühlende Prinzip, fördern. $\mathrm{Zu}$ Nahrungsmitteln, die Beken verstärken, gehören Teigwaren, Süssigkeiten, frische Milchprodukte sowie rohes Gemüse.

- Trinken von heissem Wasser morgens nach dem Aufstehen; wässrige, frische Früchte durch getrocknete Früchte ersetzen; rohes Gemüse durch gekochtes oder gegrilltes Gemüse ersetzen.

- Die körperliche Aktivität erhöhen.

- Feuchte und kalte Umgebung vermeiden.

- Nicht fasten.

\section{Resultate}

Die Patientinnen wurden während eines Jahres beobachtet. 5 Patientinnen fuhren auch nach den 6 Behandlungsmonaten mit den Verhaltensweisen fort. Eine Patientin unterbrach die Behandlung während eines akuten Rückfalls einer Zystitis, nahm dann aber anschliessend wieder an der Behandlung teil. 
Tab. 1. Harnwegsinfekte vor und nach Behandlung

\begin{tabular}{lr}
\hline Patientinnen total & 5 \\
Harnwegsinfekte/Jahr total vor & $\geq 15$ \\
$\quad$ Behandlung & $\geq 3$ \\
Pro Patientin & 5 \\
\hline Ausgewertete Patientinnen & 3 \\
Harnwegsinfekte total während & \\
$\quad$ 12 Monaten Behandlung und \\
$\quad$ Beobachtung
\end{tabular}

Während der 12 Monate der Beobachtung hatten 3 Patientinnen keinen Rückfall und waren vollständig frei von Harnwegsinfekten. Eine Patientin hatte während diesen 12 Monaten eine Harnwegsinfektion, 2 weitere Patientinnen hatten 2 Rückfälle (Tab. 1).

Weiter verminderten sich während diesen 12 Monaten folgende andere Symptome, die gemäss der Tibetischen Medizin mit «Beschwerden von kalten Nieren» zusammenhängen: Meteorismus, Flatulenz, Gefühl von Kälte in der Lumbalregion, Kältegefühl beim Betasten der Hüftregion, Neigung zu Erkältung, Blässe, Schwäche oder Müdigkeit, Dysmenorrhö und kalte Glieder (vor allem Füsse).

\section{Diskussion}

Die zwei hier beschriebenen Studien zeigen einerseits eine Wirkung von Se'Bru 5 bei dyspeptischen Beschwerden, andererseits - zusammen mit den getroffenen Ernährungs- und Verhaltensmassnahmen - eine Wirkung gegen rezidivierende Harnwegsinfekte. Eine Auswertung der in den 5 Arzneipflanzen enthaltenen Inhaltsstoffe erklärt diese Wirksamkeit (Tab. 2).
Tab. 2. Inhaltsstoffe und bekannte pharmakologische Wirkungen der Arzneipflanzen von Se'Bru 5

\begin{tabular}{|c|c|c|c|c|c|c|}
\hline Inhaltsstoffe & GSA & GW & FLP & KS & KZR & Pharmakologische Wirkung \\
\hline Ätherische Öle & & $\mathrm{X}$ & $\mathrm{X}$ & $\mathrm{X}$ & $\mathrm{X}$ & $\begin{array}{l}\text { Stimulation der Verdauungssäfte, Spasmolyse, } \\
\text { karminativ, Bakterienhemmung, Appetitanregung }\end{array}$ \\
\hline Scharfstoffe & & $\mathrm{X}$ & $\mathrm{X}$ & & & $\begin{array}{l}\text { Desensibilisierung überaktiver Schmerzrezeptoren, } \\
\text { Stimulation der Verdauungssäfte, Erhöhung der } \\
\text { gastrointestinalen Motilität, Schleimhautschutz, } \\
\text { Verminderung von Übelkeit, Appetitanregung }\end{array}$ \\
\hline Flavonoide & & $\mathrm{X}$ & & & & $\begin{array}{l}\text { Desensibilisierung überaktiver Schmerzrezeptoren, } \\
\text { Entzündungshemmung, Antioxidation }\end{array}$ \\
\hline Tannine & $\mathrm{X}$ & & & & $\mathrm{X}$ & $\begin{array}{l}\text { Entzündungshemmung, Immunmodulation, } \\
\text { Schleimhautschutz, Reizminderung }\end{array}$ \\
\hline Bitterstoffe & & $\mathrm{X}$ & & & & $\begin{array}{l}\text { Stimulation der Magensekretion, Appetitanregung, } \\
\text { Verbesserung der Nährstoffabsorption }\end{array}$ \\
\hline Schleimstoffe & & & & & $\mathrm{X}$ & Schleimhautschutz, Reizminderung \\
\hline Fruchtsäuren & $\mathrm{X}$ & & & & & $\begin{array}{l}\text { Verbesserung der Proteinverdauung, Entzündungs- } \\
\text { hemmung, Verbesserung des Immunsystems }\end{array}$ \\
\hline
\end{tabular}

GAA = Granatapfelsamen; GW = Galgantwurzel; FLP = Früchte des Langen Pfeffers;

$\mathrm{KF}=$ Kardamomsamen; KZR = Kassia-Zimtrinde.
Die Verbesserung der Gastromotilität und der Gastroprotektion, Stimulation der Verdauungssäfte, der Schleimhautschutz usw. zeigen klar die Wirksamkeit gegen Dyspepsie an.

Das im Langen Pfeffer vorhandene Piperin mit heissen und scharfen Eigenschaften scheint muskelrelaxierende Eigenschaften zu haben [3]. Diese Wirkung sowie die antibakteriellen, analgetischen, immunmodulierenden sowie schleimhautprotektiven Eigenschaften der verschiedenen Inhaltsstoffe der in Se'Bru 5 enthaltenen Arzneipflanzen sind eine plausible Erklärung für die in der Kasuistik beschriebene Wirksamkeit gegen rezidivierende Harnwegsinfekte.

2 Vennos C, Thupten T: An expanded view on the formula Se'Bru 5 in the modern context. Sowa Rigpa J 2017;7:12-17.

\section{Fazit}

Das tibetische Medikament Se'Bru 5, eine Mischung von 5 getrockneten und gemahlenen Arzneipflanzen, hat aufgrund der Datenlage die Zulassung für die Indikationen Verdauungsstörungen sowie Appetitmangel erhalten. Die vorgestellte Studie von Meier et al. [1] von 2013 bestätigt diese Wirksamkeit.

Mit dem oben beschriebenen Fallbericht öffnet sich allenfalls eine neue Indikation für das tibetische Medikament. Der Verlauf rezidivierender Harnwegsinfekte bei den 5 Patientinnen sowie die Interpretation der Wirkungen, die für die Inhaltsstoffe der 5 Arzneipflanzen von Se'Bru 5 nachgewiesen sind, lassen hoffen, dass diese Indikation in der Schweiz bald zugelassen wird.

\section{Literatur}

1 Meier R, Hengstler P, Weber F, et al: The Tibetan herbal formula Padma Digestin in functional dyspepsia: an open-label study. Forsch Komplementmed 2013;20(suppl 2):2-7.
3 Boudaka A, Wörl J, Shiina T, et al.: Involvement of TRPV1-dependent and -independent components in the regulation of vagally induced contractions in the mouse esophagus. Eur J Pharmacol 2007;556:157-165. 